\title{
Digital WATERMARKING TECHNIQUE BASED ON MULTI-RESOLUTION CURVELET TRANSFORM
}

\author{
Ranjeeta Kaushik $^{1}$, Sanjay Sharma ${ }^{2}$ and L. R. Raheja ${ }^{3}$ \\ ${ }^{1}$ Department of Computer Science and Engineering, Thapar University, Patiala, Punjab, \\ India \\ ${ }^{2}$ Department of Electronics and Communication Engineering, Thapar University, Patiala, \\ Punjab, India \\ ${ }^{3}$ Department of Navel Engineering, IIT, Kharagpur, India.
}

\begin{abstract}
In this paper an efficient \& robust non-blind watermarking technique based on multi-resolution geometric analysis named curvelet transform is proposed. Curvelet transform represent edges along curve much more efficiently than the wavelet transform and other traditional transforms. The proposed algorithm of embedding watermark in different scales in curvelet domain is implemented and the results are compared using proper metric. The visual quality of watermarked image, efficiency of data hiding and the quality of extracted watermark of curvelet domain embedding techniques with wavelet Domain at different number of decomposition levels are compared. Experimental results show that embedding in curvelet domain yields best visual quality in watermarked image, the quality of extracted watermark, robustness of the watermark and the data hiding efficiency.
\end{abstract}

\section{INTRODUCTION}

Due to popularity of internet and its increasingly easy access to digital multimedia, a number of powerful tools are available for editing of digital media without the loss of quality. So authentication and the intellectual property right of digital media is an important issue i.e to protect the intellectual property right (IPR) of the author (copyright protection). There are many solutions to this problem such as digital signature and digital watermarking. In digital watermarking we embed the watermark in the original work such that it preserves quality of watermarked data. The watermark can later be extracted for the purpose of author's identification and integrity verification. The main goal of watermarking is to hide a message $m$ in some audio or video (cover) data $d$, to obtain new data $d^{\prime}$, practically indistinguishable from $d$, by people, in such a way that an eavesdropper cannot remove or replace $m$ in $d^{\prime}$. In the present work the existing techniques of digital watermarking are briefly described and compared with respect to invisibility, efficiency and the quality of extracted watermark using appropriate metrics. The aim of the digital watermarking is to embed some specific information about the author in the form of text or image in to the original work without making it visible [1]. The purpose is not only to hide the data but prevent any modification or removal of embedded watermark. The original image is called as cover image and the output image is called as watermarked image In section II watermark system evaluation metric are presented. Wavelet transforms and the two algorithms one is embedding watermark in HL, LH frequencies and second is embedding in LL frequencies are presented in session III. The curvelet transform and proposed algorithm are described in 
section IV. The experimental results are given in section V. Section VI presents the comparison of proposed algorithm with wavelet transform and finally section VII provides the conclusion

\section{WATERMARK SYSTEM EVALUATION}

A watermarking system has a number of requirements. Obviously, different applications have different concerns; therefore, there is no set of properties that all watermarking systems have to satisfy.

\subsection{INVISIBILITY}

Embedding extra information in the original signal will cause degradation and perceptual distortion. [4]. The most common evaluation method is to compute the peak signal-to-noise ratio (PSNR) between the host and watermarked signals. PSNR is defined as follows:

$$
P S N R=10 \log _{10} \frac{255^{2}}{M S E}
$$

More PSNR means better image quality.

Where MSE (Mean Square Error) is define as

$M S E=\frac{1}{n} \sum_{i=1}^{n}(\operatorname{Im}(i)-\operatorname{Iw}(i))^{2}$

Where $\mathrm{I}_{\mathrm{m}}=$ Original image, $\mathrm{I}_{\mathrm{w}}=$ watermarked image

\subsection{EFFECTIVENESS}

Digital watermarking systems have a dependence on the input signal. Effectiveness refers to whether it is possible to detect a watermark immediately following the embedding process [6]. Although $100 \%$ effectiveness is ideal, it is often not possible to achieve such a high rate.

The quality of extracted watermark can be accessed by using Normalized Correlation between the original and extracted watermark. Normalized (NC) coefficients are defined as follow.

$N C=\frac{\sum_{m} \sum_{n}\left(\mathrm{Wo}_{\mathrm{mn}}-\overline{\mathrm{Wo}_{\mathrm{mn}}}\right)\left(\mathrm{Wx}_{\mathrm{mn}}-\overline{\mathrm{Wx}}\right)}{\sqrt{\sum_{\mathrm{m}} \sum_{\mathrm{n}}\left(\mathrm{Wo}_{\mathrm{mn}}-\overline{\mathrm{Wo}}_{\mathrm{mn}}\right)^{2}\left(\mathrm{Wx}_{\mathrm{mn}}-\overline{\mathrm{Wx}_{\mathrm{mn}}}\right)^{2}}}$

Where $\mathrm{Wo}_{\mathrm{mn}}$ is the original watermark image with $\mathrm{m}^{*} \mathrm{n}$ size, $\mathrm{Wx}_{\mathrm{mn}}$ is the extracted watermark image with $\mathrm{m}^{*} \mathrm{n}$ size.

$\overline{\mathrm{Wo}} \mathrm{o}_{\mathrm{mn}}, \overline{\mathrm{Wx}} \mathrm{x}_{\mathrm{mn}}$ Are the means of the original watermark and extracted watermark images respectively?

\subsection{EFFICIENCY}

Efficiency refers to the embedding capacity. For images, it is usually expressed in bits of information per pixel (bpp). The desired size of the watermark is application dependent. Although it is possible to embed as much information as one wants, the sacrifice is often the signal quality 
or the invisibility. A common evaluation method of the watermarking system is to plot PSNR vs. Efficiency, where one can examine both properties together.

\subsection{ROBUSTNESS}

Robustness refers to the ability for the detector to detect the watermark after signal distortion, such as format conversion, introduction of transmission channel noise and distortion due to channel gains

\subsection{SECURITY}

One of the major goals of a digital watermarking system is to protect digital content from illegal use and distribution. However, the protection is diminished if the attackers can estimate, remove, or insert a watermark [6]. Extraction processes such as Pubic, Semi Build, and Build.

\section{WAVELET TRANSFORM}

\subsection{Continuous Wavelet Transformation (CWT)}

It is defined as

$$
\mathrm{CWT}_{\mathrm{x}}^{\psi}(\tau, \mathrm{s})=\psi_{\mathrm{x}}^{\varphi}(\tau, \mathrm{s})=\frac{1}{\sqrt{|\mathrm{s}|}} \int \mathrm{x}(\mathrm{t}) \psi^{*}\left(\frac{\mathrm{t}-\tau}{\mathrm{s}}\right) \mathrm{dt}
$$

Where $\tau$ is translation, $\mathrm{s}$ is scale parameter, $\psi(\mathrm{t})$ is mother wavelet prototype for generating the other window functions.

Inverse continuous wavelet transform (ICWT) is given as follow

$$
\begin{array}{r}
x(t)=\frac{1}{c_{\varphi}^{2}} \int_{s} \int_{\tau} \Psi_{\mathrm{x}}^{\Psi}(\tau, s) \frac{1}{s^{2}} \varphi\left(\frac{t-\tau}{s}\right) d \tau d s \\
\text { Where } \quad c_{\varphi}=\left\{2 \pi \int_{-\infty}^{\infty} \frac{|\widehat{\varphi}(\xi)|^{2}}{|\xi|} d \xi\right\}^{1 / 2}<0
\end{array}
$$

Where $\hat{\varphi}(x i)$ is the FT of $\psi(t)$ equation (vi) implies that the $\hat{\varphi}(x i)=0$ which is

$$
\int \psi(t) d t=0
$$

\subsection{Discrete WAVELET TranSFORM (DWT)}

As it is well known wavelet transform provides information about frequency domain as well as time domain at same instant. Embedding of watermark in wavelet transform is relatively of better quality and robustness. Discrete wavelet transform coefficients are obtained by standard procedure in which a dyadic grid is used for scale and translation parameter [7]. Signal sequence is decomposed in to sub bands of frequencies at different levels for eg. To start with the signal will be decomposed in to two bands manly low and high ( $\mathrm{L} \& \mathrm{H}$ ) frequency i.e if $500 \mathrm{~Hz}$ is the highest frequency by passing it though a low pass and high pass filter and convoluted then the two band will contains the coefficients from 0 to $250 \mathrm{~Hz}$ and 250 to $500 \mathrm{~Hz}$ respectively. 
Thereafter each sub band will be further decomposed in to two sub bands LL1, LH1 and HL1, HH1. This procedure will continue to obtain the higher level of decomposition. As shown in Fig. 1.

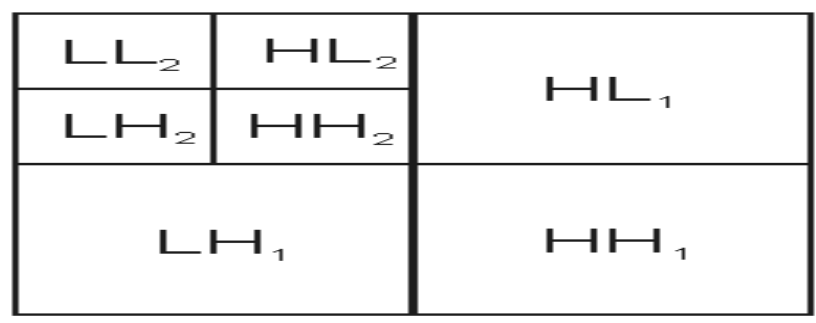

Fig 1.Wavelet Transform

\subsubsection{Algorithm For Embedding The Watermark In, LH, HL Sub Bands Of FREQUENCIES}

Embedding watermark LL, HL \& LH frequencies regions allows us to increase the robustness of our watermark; at little to no additional impact on image quality [9].In the algorithm given below we have used Haar wavelet filter and a threshold (tr) value of the frequency which is the average values of HL and LH frequencies.

\section{Algorithm}

Step 1: Read cover image and watermark image

Step 2: Resize the watermark image in to vector and set threshold i.e. $\operatorname{Tr}$

Step3: Apply 2 levels DWT to the original image. Four frequencies matrices are generated called as LL, HL, LH, and HH

Step4: if the watermark $>$ threshold $(\mathrm{Tr})$

HL coefficient value $=$ HL coefficient value + watermark

Else $\mathrm{LH}$ coefficient value $=\mathrm{LH}$ coefficient value +watermark

Step 5: Repeat step 4 until the length of watermark! $=0$

Step 6: Apply inverse wavelet transform to transform a frequency domain image in to spatial domain.

\subsubsection{Algorithm For Embedding The WATERMark IN LL Sub Bands Of FREQUENCIES}

Embedding in LL frequency band the threshold tr has been taken as zero. This is done because the LL band of frequency in general contains maximum information of the image.

\section{Algorithm}

Step 1: Read cover image and watermark image

Step 2: Apply 2 levels DWT to the original image. Four frequencies matrices are generated called as LL, HL, LH, and HH

Step3: Resize the watermark image in the size of LL matrix.

Step4: LL coefficient value $=$ LL coefficient value+ watermark 
Step 5: Repeat step 4 until the length of watermark $!=0$

Step 6: Apply inverse wavelet transform to transform a frequency domain image in to spatial domain.

\section{Curvelet Transform}

Wavelets don't describe well highly anisotropic elements and contains only fixed number of directional elements, independent of scale. Wavelet is non-geometrical and do not exploit the regularity of the edge curve.

Curvelet transform has been developed to overcome the limitations of wavelet. Though wavelet transform has been explored widely in various branches of image processing, it fails to represent objects containing randomly oriented edges and curves as it is not good at representing line singularities.[9][10]

\subsection{Continuous Curvelet Transform}

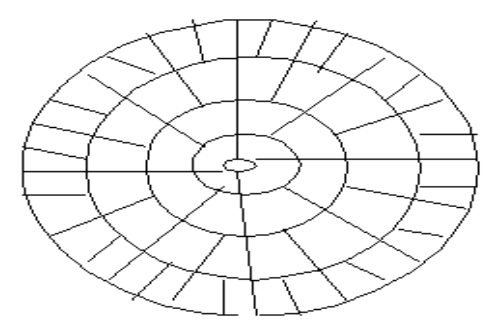

Fig. 1.1 Polar coordinates

Dilated Basic curvelet in polar coordinate.

$\widehat{\emptyset}_{j, 0,0}(r, w)=2^{-\frac{3 j}{4}} W\left(2^{-j} r\right) V_{N j}(w)$

(vii)

Where $\mathrm{W}$ and $\mathrm{V}$ are window function

$\mathrm{Nj}=$ Number of wedges in the circular ring at scale $2^{-\mathrm{j}}$

$\mathrm{V}(\mathrm{w})=\left\{\begin{array}{lc}1 & |\mathrm{w}| \leq \frac{1}{3} \\ \operatorname{Cos}\left[\frac{\pi}{2} V(3|w|-1] \frac{1}{3} \leq|w|\right. & \text { else } \\ 0 & \text { els }\end{array}\right\}$

$W(r)=\left\{\begin{array}{cc}\cos \left[\frac{\pi}{4} V(5-6 r)\right] \frac{2}{3} \leq r \leq 5 / 6 \\ \cos \left[\frac{\pi}{4} V(3 r-4)\right] & 4 / 3 \leq r \leq 5 / 3 \\ 1 & \frac{5}{6} \leq r \leq \frac{4}{3} \\ 0 & \text { else }\end{array}\right.$ 
Where $\mathrm{v}(\mathrm{x})$ is a smooth function satisfying

$$
\begin{gathered}
v(x)= \begin{cases}0 & x \leq 0 \\
1 & x \geq 1\end{cases} \\
v(x)+v(1-x)=1
\end{gathered}
$$

$v(x)$, where for the tiling of a circular ring in to $\mathrm{N}$ wedges, Where $\mathrm{N}$ is arbitrary positive integer. We need to $2 \pi$ periodic non negative window with support inside $[-2 \pi / \mathrm{N}$, a $\pi / \mathrm{N})$ such that [15][16]

$\sum_{l=0}^{N-1} V_{N}\left(w-2 \pi \frac{1}{N}\right)=1 \quad w \in\{0,2 \pi)$

The sum of squared rotation curveletfunction depends only on $w\left(2^{-\mathrm{j}} \mathrm{r}\right)$ follows that

$\sum_{l=0}^{N_{j-1}}\left|2^{\frac{3 j}{4}} \widehat{\emptyset}_{j, 0,0}\left(r, w-\frac{2 \pi l}{N_{j}}\right)^{2}\right|=\left|w\left(2^{-j} r\right)^{2} \sum_{l=0}^{N_{j-1}} V_{N}\left(w-\frac{2 \pi l}{N_{j}}\right)\right|=\left|w\left(2^{-j} r\right)\right|^{2}$

$c_{j, k, l}(f)=<f, \emptyset_{j, k, l}$ are called curvelet transform

$c_{j, k, l}(f)=\int_{R^{2}} f(X) \overline{\emptyset_{j, k, l}(X)} \mathrm{dx}=\int \hat{f}(\xi) \overline{\hat{\phi}_{j, k, l}}(\xi) d \xi$

$=\int \hat{f}(\xi) \overline{\hat{\phi}_{j, 0,0}}\left(R_{\theta, 12 j, l} \xi\right) e^{i\left(b_{k}^{j, l}, \xi\right)} d \xi$

\subsection{Digital Curvelet Transform}

Digital curvelet transform is different from continuous as its take input as Cartesian Array instead of polar tilling, its make virtual concentric squares instead of circle [2].

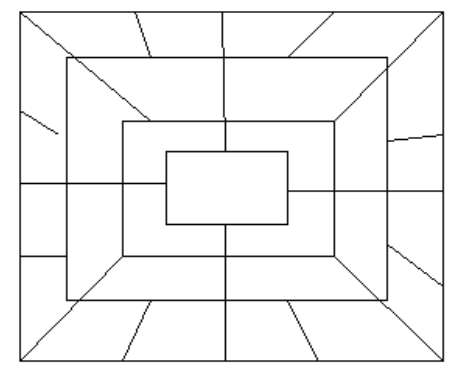

Fig. 1.2 Cartesian array on input

Basic curvelet according to new tiling rotation is replaced by shearing.

$\widetilde{\emptyset_{J, 0,0}}(\xi)=2^{-3 j / 4} W\left(2^{-j} \xi_{1}\right) V\left(\frac{2^{-\frac{j}{2}} \xi_{2}}{\xi_{1}}\right)$

The adopted basic curvelet $\emptyset_{j, o, o}$ determine the frequencies in the trapezoid

$\left\{(\xi 1, \xi 2): 2^{j-1} \leq \xi 1 \leq 2^{j+1},-2^{\left\lfloor\frac{j}{2\rfloor}\right.} \cdot \frac{2}{3} \leq \frac{\xi 1}{\xi 2} \leq 2^{-\frac{j}{2}} \cdot \frac{2}{3}\right\}$ 
In order to replace rotation of curvelet elements by shearing in the new grid, there is need to consider the cones of each side separately instead of equi-spaced angles, we define as of equispaced slopes for all cones

Where $l=-2^{\lfloor j / 2\rfloor}+1 \ldots \ldots \ldots .2^{\lfloor j / 2\rfloor}-1$

$$
\tan \theta_{j, l}=l 2^{-\lfloor j / 2\rfloor}
$$

Now the curvelet like function be given by

$\varphi_{j, k, l}(x)=\varphi_{j, 0,0}\left(S_{q, j, l}^{t}\left(x-b_{k}^{j, l}\right)\right)(\mathrm{xv})$

With shear matrix $\quad S_{q=}\left[\begin{array}{cc}1 & 0 \\ \tan \theta & 1\end{array}\right]$

So the digital curvelet transform

$$
b_{k}^{j, l}=S_{q, j, l}^{-t}\left(k_{1} 2^{-j}, k_{2} 2^{-j / 2}\right.
$$

$\left.\left.\varphi_{j, k, l}(\xi)=e^{-i(} b_{k}^{j, l}, \xi\right) \varphi_{j, 0,0}\left(S_{q, j, l}^{-t}, \xi\right)=e^{-i(} b_{k}^{j, l}, \xi\right) 2^{-3 j / 4} W\left(2^{-j} \xi_{1}\right) V\left(\frac{2^{-\frac{j}{2}} \xi_{2}}{\xi_{1}}\right)$

We find the Cartesian counterpart of the coefficients in equation by

$$
\begin{aligned}
& \tilde{c}_{j, k, l}(f)=<f, \widehat{\phi}_{j, k, l}>=\int_{\mathbb{R}^{2}} \hat{f}(\xi) \hat{\phi}_{j, 0,0}\left(s_{\theta, j, l}^{-1} \xi\right) e^{i\left(b_{k}^{-j, l}, \xi\right)} d \xi \\
& =\int_{\mathbb{R}^{2}} \hat{f}\left(S_{\theta, j, l} \xi\right) \hat{\phi}_{j, 0,0}(\xi) e^{i\left(k_{j}, \xi\right)} d \xi \\
& \operatorname{With}_{j}=\left(k_{1} 2^{-j}, k_{2} 2^{-\frac{j}{2}}\right),\left(k_{1}, k_{1}\right)
\end{aligned}
$$

\subsection{ALgORITHM}

1. Compute the Fourier transfrom of $f$ by means of a $2 D$ FFT. Let $\tilde{S}(x)=s\left(x_{1}\right) s\left(x_{2}\right)$ with $s\left(x_{1}\right)=\left(1-\left|x_{1}\right|\right) \chi_{|-1,1|}\left(x_{1}\right)[2]$

$$
\begin{aligned}
& f(x)=\sum_{n_{1}=0}^{N-1} \sum_{n_{2}=0}^{N-1} f\left(\frac{n_{1}}{N}, \frac{n_{2}}{N}\right) \tilde{s}\left(N x_{1}-n_{1}, N x_{2}-n_{2}\right) \\
& \hat{f}(\xi)=\sum_{n_{1}=0}^{N-1} \sum_{n_{2}=0}^{N-1} f\left(\frac{n_{1}}{N}, \frac{n_{2}}{N}\right) e^{-\frac{i\left(n_{1} \xi_{1}+n_{2} \xi_{2}\right)}{N} \hat{s}}\left(\frac{\xi}{N}\right)
\end{aligned}
$$

The 2D FFT of length $\mathrm{N}$ gives us the samples from [14][15]

2. Computer $\hat{f}\left(S_{\theta, j, l} \xi\right)$ by interpolation

3. Compute the product $\hat{f}\left(S_{\theta, j, l} \xi\right) \hat{\phi}_{j, 0,0}(\xi)$

4. Apply the inverse 2D FFT in order to obtain the discrete coefficients that is an approximation of the coefficients. 


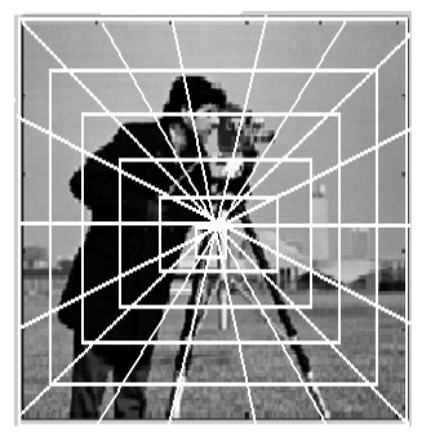

Fig. 2 Curvelet Transform

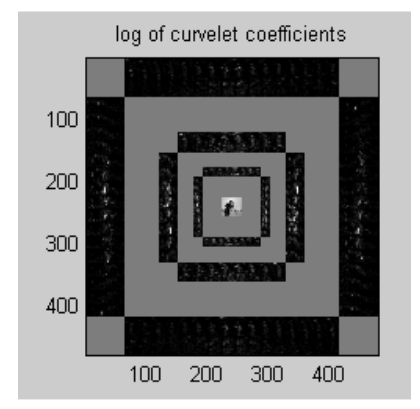

Fig. 2.1 Coefficients of curvelet

The above fig gives the curvelet coefficients. The low frequency coefficients are stored at the centre of the display. The Cartesian concentric coronae show the coefficients at different scale. The outer coronae correspond to higher frequencies. There are four strips associated to each corona corresponding to the four cardinal points; these are further subdivided in angular panels. Each panel represents coefficients at a specified scale and along the orientation suggestion by the position of the panel.

\subsection{WATERMARK EMBEDding Algorithm}

1. Apply USFFT digital curvelet to the $n * m$ size cover image, get curvelet coefficients matrix C.

2. Read the watermark and covert in into binary image then resize the watermark in some small size.

3. Select the scale and oriantation for embedding let in this algo we use scale 2 and orientation due to the advantages of low frequencies

4. Select the curvelet coefficients to embed a bit of watermark in the curvelet coefficents with 2 scale as $\mathrm{C}^{\prime}(\mathrm{j}, \mathrm{l}, \mathrm{k})=\mathrm{C}(\mathrm{j}, \mathrm{l} . \mathrm{k})+\mathrm{W}[\mathrm{k}]$;

5. Do Inverse curvelet trasform to obtain the watermarked image i.e C'

\section{EXPERIMENT RESULTS}

We used a gray level cameraman image for experiment. The cover image is size of $256 * 256$ and the watermark image is binary copyright image. Fig 3 and Fig 4 shows the cover image and 
watermark image respectively. Fig 5 to Fig 7 shows the results of above discussed algorithms. Fig 5 \& Fig 6 show embedding in HL \&LH frequencies, LL frequencies in wavelet transform respectively. Fig 7 shows the embedding of the same in curvelet transform. Fig 8 to fig 10 presents the extracted watermark of above discussed techniques

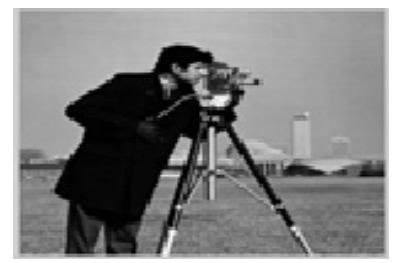

Fig. 3 Cover Image

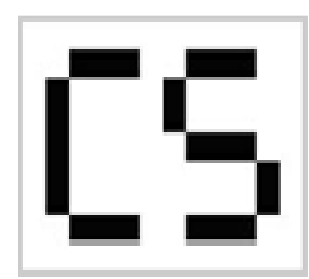

Fig. 4 Watermark Image

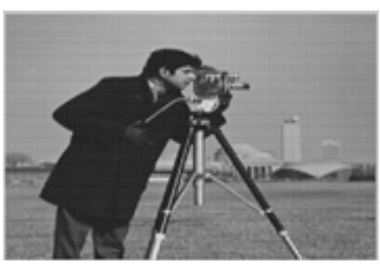

Fig. 5 Wavelet Transformation embedding in HL \& LH Frequencies PSNR $=69.014$

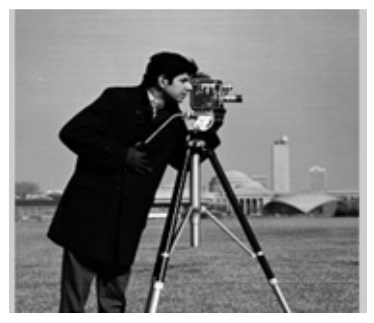

Fig. 6 wavelet transform in LL frequencies PSNR $=72.0251$

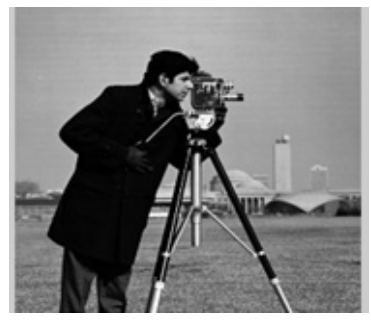

Fig. 7 Curvelet Domain in 2 scale PSNR $=77.0099$ 


\subsection{RESUlts Of EXTRACTED WATERMARK}

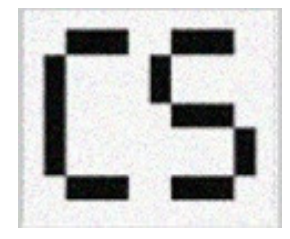

Fig. 8 wavelet in HL LH Freq PSNR =57.7729

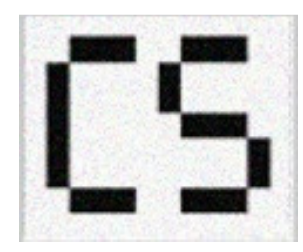

Fig.9 wavelet in LL Frequencies PSNR $=53.9633$

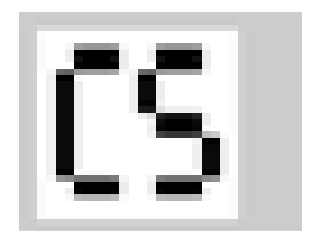

Fig.10 Curvelet Transform PSNR= 64.2457

\subsection{INVISIBILITY AND EFFICIENCY TEST}

Table 1.1 shows the PSNR values, NC values and the embedding capacity of the watermarked image at different scales in curvelet domain. From the table it very much clear that the embedding in lower scales give better invisibility without significantly affecting the PSNR values.NC of watermarked image is 1 for all the scales confirming the quality of extracted watermark. Further all scales have the same efficiency with regard to data hiding capacity as because the watermark of the size of the cover image can embedded with significantly effect on PSNR of watermarked image. So efficiency of data hiding is very high.

\begin{tabular}{|c|c|c|c|c|c|}
\hline $\begin{array}{l}\text { Sr. } \\
\text { No }\end{array}$ & Domain & $\begin{array}{l}\text { Selected } \\
\text { Coefficients }\end{array}$ & $\begin{array}{l}\text { PSNR of } \\
\text { Watermarked } \\
\text { image }(\mathrm{dB})\end{array}$ & $\begin{array}{l}\text { NC of } \\
\text { Watermarked } \\
\text { image }\end{array}$ & $\begin{array}{l}\text { Embedding } \\
\text { Efficiency (no. of } \\
\text { bits) }\end{array}$ \\
\hline 1 & \multirow{5}{*}{ Curvelet } & Scale 2 & 77.0099 & 1.000 & $256^{*} 256$ \\
\hline 2 & & Scale 3 & 76.9622 & 1.000 & $256^{*} 256$ \\
\hline 3 & & Scale 4 & 76.9621 & 1.000 & $256^{*} 256$ \\
\hline \multirow[t]{2}{*}{4} & & Scale 5 & 76.9620 & 1.000 & $256^{*} 256$ \\
\hline & & Scale 6 & 76.9466 & 1.000 & $256^{*} 256$ \\
\hline
\end{tabular}

Table 1.1 Comparision Of Perposed Algorithm Embedding In Different Scales

\subsection{ROBUSTNESS TEST}

Robustness is the most important metric for watermark system evaluation. Robustness is the ability of watermark detection after some attacks or distortion of image. In table 1.2 the PSNR is employed to evaluate the quality of watermark image after different types of attacks on different scales in curvelet domain. Table 1.3 gives the normalize correlation (NC) between the original watermark and extracted watermark image after different types of attacks. Attacks are like adding of noise \&filtering of image. It can be seen from both table 1.2 and 1.3 that the embedding in 
scale 6 in curvelet domain gives better robustness against the pepper \& salt noise, Gaussian noise, low pass filtering and median filtering compared to lower scales.

\begin{tabular}{|c|l|l|l|l|l|l|}
\hline Sr. & \multirow{2}{*}{$\begin{array}{c}\text { No. } \\
\text { Domain }\end{array}$} & $\begin{array}{l}\text { Selected } \\
\text { Coefficients }\end{array}$ & $\begin{array}{l}\text { PSNR of } \\
\text { Extracted } \\
\text { image(dB) } \\
\text { Adding } \\
\text { noise(Paper } \\
\text { salt) }\end{array}$ & $\begin{array}{l}\text { PSNR of } \\
\text { Extracted } \\
\text { image(dB) } \\
\text { Adding } \\
\text { noise(Gaussian) }\end{array}$ & $\begin{array}{l}\text { Filtered } \\
\text { image }\end{array}$ & $\begin{array}{l}\text { Median } \\
\text { Filter }\end{array}$ \\
\hline \multirow{3}{*}{1} & \multirow{2}{*}{\begin{tabular}{c} 
Curvelet \\
\cline { 3 - 7 }
\end{tabular}} & Scale 6 & 31.9855 & 31.9863 & 21.3016 & 33.6071 \\
\cline { 3 - 7 } & Scale 5 & 31.9859 & 31.9808 & 21.3016 & 33.6071 \\
\hline
\end{tabular}

Table 1.2 Psnr Value Of Extracted Watermark After Some Image Processing Operations

\begin{tabular}{|l|l|l|l|l|l|l|}
\hline \multirow{2}{*}{$\begin{array}{l}\text { Sr. } \\
\text { No. }\end{array}$} & Domain & $\begin{array}{l}\text { Selected } \\
\text { Coefficients }\end{array}$ & $\begin{array}{l}\text { NC Extracted } \\
\text { image } \\
\text { Adding } \\
\text { noise(Paper } \\
\text { salt) }\end{array}$ & $\begin{array}{l}\text { NC Extracted } \\
\text { image Adding } \\
\text { noise(Gaussian) }\end{array}$ & $\begin{array}{l}\text { NC } \\
\text { Filtered } \\
\text { image }\end{array}$ & $\begin{array}{l}\text { Median } \\
\text { Filter }\end{array}$ \\
\hline \multirow{2}{*}{1} & \multirow{2}{*}{ Curvelet } & Scale 6 & 0.9702 & 0.985 & 0.927 & 0.98 \\
\cline { 3 - 7 } & Scale 5 & 0.9543 & 0.9683 & 0.893 & 0.91 \\
\cline { 2 - 7 } & Scale 4 & 0.8828 & 0.909 & 0.87 & 0.821 \\
\hline
\end{tabular}

Table 1.3 NC Value of Extracted Watermark after Some Image Processing Operations

Table 1.4 contains the NC values of extracted watermark of pepper \& salt noise attack at different density parameter. Table 1.5 contains the NC value of extracted watermark image of Gaussian noise at different standard variance with same mean. It can be seen from both these tables that embedding in curvelet domain in $6^{\text {th }}$ scale gives the better robustness even at higher levels of noise.

\begin{tabular}{|c|c|c|c|c|}
\hline S.no & Domain & Selected Coefficients & $\begin{array}{c}\text { Noise Density } \\
\text { Parameter }\end{array}$ & $\begin{array}{l}\text { NC value of } \\
\text { extracted } \\
\text { watermark }\end{array}$ \\
\hline \multirow{7}{*}{1} & \multirow{7}{*}{ CurveletTransform } & \multirow{7}{*}{ Scale 6} & 0.01 & 0.9702 \\
\hline & & & 0.02 & 0.9567 \\
\hline & & & 0.03 & 0.9298 \\
\hline & & & 0.05 & 0.9074 \\
\hline & & & 0.07 & 0.8872 \\
\hline & & & 0.09 & 0.8761 \\
\hline & & & 0.1 & 0.8291 \\
\hline
\end{tabular}

Table 1.4 NC Value of Extracted Watermark after Adding Pepper \& Salt Noise 


\begin{tabular}{|c|c|c|c|c|}
\hline S.no & Domain & $\begin{array}{l}\text { Selected } \\
\text { Coefficients }\end{array}$ & $\begin{array}{c}\text { Standard Variance of } \\
\text { Gaussian Noises }\end{array}$ & $\begin{array}{c}\text { NC value } \\
\text { extracted } \\
\text { watermark and } \\
\text { original } \\
\text { watermark }\end{array}$ \\
\hline \multirow{7}{*}{1} & \multirow{7}{*}{$\begin{array}{l}\text { Curvelet } \\
\text { Transform }\end{array}$} & \multirow{7}{*}{ Scale 6} & 0.001 & 0.985 \\
\hline & & & 1 & 0.9587 \\
\hline & & & 3 & 0.936 \\
\hline & & & 5 & 0.896 \\
\hline & & & 6 & 0.877 \\
\hline & & & 10 & 0.856 \\
\hline & & & 20 & 0.813 \\
\hline
\end{tabular}

Table 1.5 NC Value of Extracted Watermark after Adding Gaussian Noise

\subsection{ANALYSIS}

In order to analyze different embedding techniques we compared our proposed scheme with wavelet transform technique at different levels. Using out algorithm we have embedded the watermark in HL domain and a copy of same in LH domain. In second algorithm embedding is performed in LL frequencies only. Table 1.6 contains the PSNR of watermarked image, PSNR of extracted watermark and the embedding capacity of the domain. From the table it clear that the embedding in LL frequencies give better invisibility of watermarked image but the efficiency of data hiding reduces. The Maximum PSNR of watermarked image is achieved in $3^{\text {rd }}$ level of decomposition i.e. embedding in LL frequencies .By comparing the table 1.1 and 1.6 it observed that the embedding in curvelet domain at any scale gives the better PSNR of watermarked image and better information hiding capacity. Further, PSNR of watermarked image at any scale is more than the any decomposition level of wavelet domain confirming the superiority of curvelet domain.

\begin{tabular}{|c|c|c|c|c|c|}
\hline Technique & Domain & $\begin{array}{l}\text { Level } \\
\text { DWT }\end{array}$ & $\begin{array}{l}\text { PSNR of } \\
\text { watermarked(d } \\
\text { B) }\end{array}$ & $\begin{array}{l}\text { PSNR of } \\
\text { Extracted } \\
\text { watermark }(\mathrm{dB})\end{array}$ & $\begin{array}{l}\text { Embedding } \\
\text { Efficiency }\end{array}$ \\
\hline \multirow{6}{*}{$\begin{array}{l}\text { Wavelet } \\
\text { transform }\end{array}$} & \multirow[t]{3}{*}{$\begin{array}{l}\text { HL \& LH } \\
\text { freq. }\end{array}$} & $1 \mathrm{DWT}$ & 57.0287 & 56.1278 & $\begin{array}{l}\left(128^{*} 128\right) \&\left(128^{*} 1\right. \\
28)\end{array}$ \\
\hline & & 2 DWT & 63.0363 & 56.8453 & $\left(64^{*} 64\right) \&\left(64^{*} 64\right)$ \\
\hline & & 3 DWT & 69.0148 & 57.7729 & $\begin{array}{l}\left(128^{*} 128\right) \&\left(128^{*} 1\right. \\
28)\end{array}$ \\
\hline & \multirow[t]{3}{*}{ LL freq. } & 1 DWT & 60.0390 & 54.0184 & $128^{*} 128$ \\
\hline & & 2 DWT & 66.0466 & 54.0054 & $64^{*} 64$ \\
\hline & & 3 DWT & 72.0251 & 53.9633 & $64 * 64$ \\
\hline
\end{tabular}

Table 1.6 Psnr Value Of Watermarked Image, Extracted Watermark \& Embedding Efficiency Of Different Decomposion Level In Wevelet Transform Domain

\section{CONCLUSION}

The curvelet transform a technique is used for embedding the watermark has been analyze with respect to invisibility, efficiency- data hiding capacity, effectiveness and robustness. It has been concluded that lower scales in curvelet domain give better invisibility without affecting the data hiding efficiency significantly. With addition of noise as attack it is found that higher scales give more robustness along with good PSNR value of watermarked image. The results have also been compared with wavelet transform technique .It shows that curvelet domain at any scale gives the better PSNR of watermarked image and better information hiding capacity. 
International Journal in Foundations of Computer Science \& Technology (IJFCST) Vol.7, No.2, March 2017

\section{REFERENCES}

[1] R. Wolfgang and E. Delp, " A watermark for digital images," Proceeding of IEEE International Conference on Image Processing, Vol. 2, pp.319-222, 1996.

[2] Jianwei MA, GerlindPlonka "A review of curvelet and recent applications" pp. 1-29

[3] C. T. Hsu and J. L. Wu, "Hidden digital watermarks in images," IEEE Transactions on Images Processing, Vo1.8, pp.58-68, Jan, 1999.

[4] P. C. Su, C. C. J. Kuo, and H. J. M Wang, "Blind digital watermarking for cartoon and map images," in Proceedings of SPIE Security and watermarking of multimedia contents, vol. 3657, pp.296-306, January 1999

[5] R. Singh. "Emerging technologies that will change the world: Digital rights management". MIT Technology Review,2001.

[6] I. J. Cox, M. L. Miller, and J. A. Bloom, “ Digital Watermarking”. San Diego, CA, USA: Academic Press, 2002.

[7] P. Tao, A. M. Eskicioglu, "A Robust Multiple Watermarking Scheme in the Discrete Wavelet Transform Domain”, Optics East 2004, Internet Multimedia Management Systems V Conference, Philadelphia, PA, October 25-28, 2004.

[8] Parameswaran, L. "Content Dependent Image Signature for Authentication Using Wavelets", Proceedings of NCIS, Karunya Deemed University, Coimbatore, Nov.2005.

[9] Emmanuel J. Candes, and David L. Donoho, "New Tight Frames of Curvelets and Optimal Representations of Objects with Piecewise C2 Singularities", Communication on Pureand Applied Mathematics, Vol.57, No.2, 2004, pp.219-266.

[10] E. Cand'es and D. Donoho, "Curvelets: A Surprisingly Effective Nonadaptive Representation of Objects with Edges", In C. Rabut A. Cohen and L. L. Schumaker, editors, Curves and Surface, Vanderbilt University Press, Nashville, TN. 2000, pp. 105---120.

[11] H. Y. Leung, L. M. Cheng and L. L. Cheng. "A Robust Watermarking Scheme Using Selective CurveletCoeffi-cients," International Conference on IntelligientInforma-tionHiding and Multimedia Signal Processing, Harbin, August 2008, pp. 465-468

[12] Minh N. Do, and Martin Vetterli, “The Finite Ridgelet Transform for Image Representation”, IEEE Transactions on Image Processing, Vol.12, No.1, 2003, pp. 16-28.

[13] I. J. Cox, J. Kilian, T. Leighton, and T. Shamoon, "Secure spread spectrum watermarking for multimedia", IEEE Transactions on Image Processing, Vol. 6, No. 12, 1997, pp. 1673-1687.

[14] J. P. Shi and Z. J. Zhai, "Curvelet Transform for Image Authentication," Rough Set and Knowledge Technology, LNAI 4062, 2006, pp. 659-664.

[15] Emmanuel J. Candes, and David L. Donoho, "New Tight Frames of Curvelets and Optimal Representations of Objectswith Piecewise C2 Singularities", Communication on Pureand Applied Mathematics, Vol.57, No.2, 2004, pp.219-266.

[16] E. Cand'es and D. Donoho, "Curvelets: A SurprisinglyEffectiveNonadaptive Representation of Objects withEdges", In C. Rabut A. Cohen and L. L. Schumaker, editors,Curves and Surface, Vanderbilt University Press, Nashville,TN. 2000, pp. 105---120. 


\section{Authors}

Er. Ranjeetapursuing Ph.D.in Computer Science and Engineering department from Thapar University, Patiala. Working as Associate professor in Chandigarh Engineering College, Landran, Mohali, Punjab. She is having 12 years of teaching experience. She is having 13 national, international and conferences publications. Her area of research is Watermarking in curvelet domain.

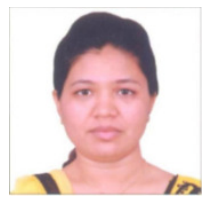

Dr. Sanjay Sharma is currently working as Professor and Head of the department in Electronics and Communication Engineering of Thapar University, India. He has done his B. Tech in ECE from REC in 1993, Jalandhar, M. E. in ECE from TTTI, Chandigarh in 2001 and Ph.D. from PTU, Jalandhar in 2006. He has completed all his education with honors. He has published many papers in various journals and conferences of international repute. He has to his credit the implementation of research projects worth

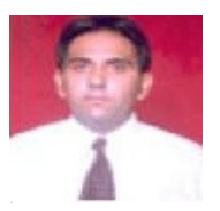
12000 USD. His main interests are VLSI Signal Processing, Wireless System Design using Reconfigurable Hardware, Digital communication, Channel Coding etc.

Dr. Lajpat R. Raheja Completed his Ph.D. in Applied Mathematics, from IIT Kharagpur, West Bengal. He has more than 30 years of teaching and research experience. He worked in number of research projects in field of computer sciences, mechanical and naval engineering. $\mathrm{He}$ is recipient of 3 internal awards Alexander von Humboldt Fellowship, West Germany, March1971-June1972, Research Scientist, InstitutfürSchiffbau, University of Hamburg, Hamburg, West Germany, Jan.1985-Jan. 1987 and Exchange Program, Department of Naval Architecture, University of Zagreb, Yugoslavia, April-June, 1979. 